PROCEEDINGS OF THE

AMERICAN MATHEMATICAL SOCIETY

Volume 133, Number 3, Pages 809-813

S 0002-9939(04)07661-0

Article electronically published on September 20, 2004

\title{
PLANAR WULFF SHAPE IS UNIQUE EQUILIBRIUM
}

\author{
FRANK MORGAN
}

(Communicated by David Preiss)

\begin{abstract}
In $\mathbf{R}^{2}$, for any norm, an immersed closed rectifiable curve in equilibrium for fixed area must be the Wulff shape (possibly with multiplicity).
\end{abstract}

\section{INTRODUCTION}

The surface energy density of a crystal in $\mathbf{R}^{n}$ is generally modeled by a norm applied to its tangent plane or unit normal ([T], [M3, Chapt. 10]). For a given volume, the Wulff shape provides the unique energy minimizer. For example, for the isotropic Euclidean norm, the Wulff shape is a round ball. For the $l^{1}$ norm $\Phi\left(\left(x_{i}\right)\right)=\sum\left|x_{i}\right|$, the Wulff shape is a cube. Our main Theorem 3.3 proves that

$$
\text { a planar equilibrium must be the Wulff shape }
$$

(possibly with multiplicity if nonembedded curves are admitted). If the norm were smooth and uniformly convex, uniqueness would follow from constant generalized curvature (see [M3, 10.4]). Our general case requires an understanding of variations where the norm is not smooth and the first variation is no longer a linear functional of the initial velocity $\mathbf{v}$.

In higher dimensions the result fails even for the isotropic Euclidean norm (area), as shown by the smooth, compact constant-mean-curvature immersions of Wente $\mathrm{W}$ ] and Kapouleas [K1, [K2]. For a new example for a "hexagonal" norm, see M2].

Nevertheless, Bennet Palmer $\mathrm{P}$ has proved that a stable equilibrium smoothly immersed hypersurface in $\mathbf{R}^{n}$ must be the Wulff shape if the norm is smooth and uniformly convex. Palmer followed the pioneering work of Barbosa and do Carmo $[\mathrm{BdC}]$ on the isotropic Euclidean norm. It is an open question whether an embedded equilibrium must be the Wulff shape, although this holds for the Euclidean norm by Aleksandrov reflection $\underline{\mathrm{A}}$.

1.1. The proof. Variational arguments prove that an equilibrium curve $C$ is locally embedded and convex. The main result then follows from Proposition 3.1, which gives a nice formula for the vector displacement between points where the norm is differentiable at a supporting tangent vector. To prove Proposition 3.1 at a point where $C$ is differentiable too, one considers a variation $\mathbf{v}$ which is constant between the points and goes to 0 in a small neighborhood of the points. At a point $p$ where $C$ is not differentiable and hence the one-sided tangents $\mathbf{T}^{-}, \mathbf{T}^{+}$are distinct, $\mathbf{v}$

Received by the editors March 30, 2003 and, in revised form, November 3, 2003.

2000 Mathematics Subject Classification. Primary 49K99.

(C)2004 Frank Morgan 
picks up a term in the $\mathbf{T}^{-}$direction just before $p$ and a term in the $\mathbf{T}^{+}$direction just after $p$.

\section{NORMS AND FIRST VARIATION}

2.1. Definitions (see [M3, Chapt. 10]). Let $\Phi$ be a (continuous) norm on $\mathbf{R}^{2}$. Let $C: \mathbf{S}^{1} \rightarrow \mathbf{R}^{2}$ be a (connected) closed rectifiable curve. Define

$$
\Phi(C)=\int \Phi\left(C^{\prime}(s)\right) d s .
$$

The curve $C$ encloses a well-defined area $A \in \mathbf{R}$, counting orientation and multiplicity. For a given area $A$, the minimum $\Phi(C)$ is provided uniquely by a scaling of the Wulff shape $W$, a rotation by 90 degrees of the unit ball $W^{\perp}$ for the norm dual to $\Phi$ :

$$
W^{\perp}=\{x: x \bullet T \leq 1 \text { if } \Phi(T) \leq 1\} .
$$

We say that $C_{0}$ is in equilibrium if for all smooth flows

$$
C_{t}(s)=C_{0}(s)+f(t, s) \quad(t \geq 0)
$$

preserving area, the derivative of $\Phi\left(C_{t}\right)$ is initially nonnegative. This one-sided derivative of $\Phi\left(C_{t}\right)$, called the first variation of $\Phi$, depends only on the initial velocity function $\mathbf{v}(s)=\left.\frac{\partial f}{\partial t}\right|_{t=0}$ and is denoted by $\delta^{1}(\mathbf{v})$. Indeed, differentiating (1) yields

$$
\delta^{1}(\mathbf{v})=\int D \Phi\left(C_{0}^{\prime}\right)\left(\mathbf{v}^{\prime}\right) d s
$$

where the integrand denotes the directional derivative at $C_{0}^{\prime}$ in the direction $\mathbf{v}^{\prime}$, which exists even if $\Phi$ is not differentiable, as long as $\Phi$ is convex and $\mathbf{v}^{\prime}$ exists. In general $\delta^{1}$ is homogeneous and $\delta^{1}(\mathbf{v}+\mathbf{w}) \leq \delta^{1}(\mathbf{v})+\delta^{1}(\mathbf{w})$. If $\Phi$ is differentiable (away from 0), then $\delta^{1}$ is linear. If $\Phi$ is differentiable at $\mathbf{T}_{0}$, then its directional derivatives are continuous at $\mathbf{T}_{0}$. Indeed, if $D \Phi\left(\mathbf{T}_{0}\right)(-\mathbf{a})=-D \Phi\left(\mathbf{T}_{0}\right)(\mathbf{a})$, then $D \Phi(\mathbf{T})(\mathbf{a})$ is continuous at $\mathbf{T}_{0}$. In particular, $D \Phi(\mathbf{T})\left(\mathbf{T}_{0}\right)$ is continuous at $\mathbf{T}_{0}$.

The general nonlinearity of the first variation $\delta^{1}$ is the reason for defining equilibrium as nonnegative rather than zero first variation. If $\mathbf{v}$ and $\mathbf{w}$ denote small congruent smooth bumps on the top of a square Wulff shape, then $\delta^{1}(\mathbf{v}-\mathbf{w})$ and $\delta^{1}(-\mathbf{v}+\mathbf{w})$ are both positive.

2.2. Proposition. A closed rectifiable curve $C$ is in equilibrium if and only if for any smooth (or Lipschitz) variation vector field $\mathbf{v}$ with $d A / d t=0, \delta^{1}(\mathbf{v})$ is nonnegative.

Proof. First we consider smooth variations. It suffices to show that any such $\mathbf{v}$ extends to an area-preserving flow. The problem is that $\mathbf{v}$ itself may change area to higher order. If $\mathbf{v}$ is transverse to $C$ at a point $p$ of differentiability of $C$, so that locally $d A / d t \neq 0$, smoothly varying in time the magnitude of $\mathbf{v}$ near $p$ yields the desired flow. Otherwise $\mathbf{v}$ is everywhere tangent to $C, C$ is smooth where $\mathbf{v}$ is nonzero, and $\mathbf{v}$ extends to a flow of $C$ along itself.

Lipschitz variations are admissible by approximation.

2.3. Proposition. Let $\Phi$ be a norm on $\mathbf{R}^{2}$. A closed rectifiable curve $C$ is in equilibrium if and only if for some real $\lambda$, the first variation of $\Phi-\lambda A$ is nonnegative for all smooth (or Lipschitz) variations $\mathbf{v}$. Such a $\lambda$ is unique and nonzero, the generalized scalar curvature. If the enclosed area is positive, so is $\lambda$. 
Remarks. Propositions 2.2 and 2.3 hold for curves with boundary, except that $\lambda$ need not be unique or nonzero. If $\Phi$ is smooth and uniformly convex, then $C$ is smooth and $|\lambda|$ is the magnitude of the generalized curvature vector $D^{2} \Phi(\boldsymbol{\kappa})$ M3 10.4]. (By [M3 10.4], $D^{2} \Phi(\boldsymbol{\kappa})$ is essentially constant with magnitude $|\lambda|$. Since $\Phi$ is uniformly convex, $D^{2} \Phi$ is smoothly invertible, and $\boldsymbol{\kappa}$ is essentially a smooth function of the unit tangent, so that $C$ is smooth.)

Proof of Proposition 2.3. Lipschitz and smooth variations are equivalent by approximation.

If the condition holds, then in particular for variations with $d A / d t=0$, the first variation of $\Phi$ equals the first variation of $\Phi-\lambda A$ and is nonnegative, the definition of equilibrium.

Conversely, let $C$ be in equilibrium. Let $\mathbf{v}_{0}$ be the variation vector field of unit homothetic expansion about a convenient center. Then for $t$ positive or negative the initial rates of change of the cost $\Phi$ and the area $A$ satisfy

$$
d \Phi / d t=\Phi(C)>0, \quad d A / d t=2 A .
$$

Since $C$ is in equilibrium, $A \neq 0$. Let $\lambda=\Phi(C) / 2 A$, so that for $\Phi-\lambda A$,

$$
\delta^{1}\left(\mathbf{v}_{0}\right)=\delta^{1}\left(-\mathbf{v}_{0}\right)=0 .
$$

This is the only possible choice for $\lambda$, and $\lambda$ is nonzero, positive if $A$ is positive. Now given any variation $\mathbf{v}$, choose $\alpha$ such that for $\mathbf{v}-\alpha \mathbf{v}_{0}, d A / d t$ initially vanishes, so that the first variation of $\Phi-\lambda A$ equals the first variation of $\Phi$ alone. Then by Proposition 2.2,

$$
0 \leq \delta^{1}\left(\mathbf{v}-\alpha \mathbf{v}_{0}\right) \leq \delta^{1}(\mathbf{v})+\delta^{1}\left(-\alpha \mathbf{v}_{0}\right)=\delta^{1}(\mathbf{v}),
$$

as desired.

\section{WULFF SHAPE IS UNIQUE EQUILIBRIUM}

Theorem 3.3, the main result of this paper, shows that in the plane the Wulff shape is essentially the unique equilibrium. Proposition 3.1 gives a nice characterization of distances in equilibrium curves.

3.1. Proposition. Let $C$ be a connected, locally convex equilibrium rectifiable curve with constant curvature $\lambda$ for a norm $\Phi$. Consider interior points $p_{1}, p_{2}$ with unit tangents $\mathbf{T}_{1}, \mathbf{T}_{2}$ to supporting lines where $\Phi$ is differentiable. Then

$$
\lambda\left(p_{2}-p_{1}\right)^{\perp}=D \Phi\left(\mathbf{T}_{2}\right)-D \Phi\left(\mathbf{T}_{1}\right),
$$

where $\perp$ denotes clockwise rotation through 90 degrees.

3.2. Remarks. If $\lambda=1$ and $\Phi$ is replaced by the associated norm $\Psi$ on normal vectors, for a convenient choice of origin, then (4) takes the simple form

$$
p=D \Psi(\mathbf{n}) .
$$

The equation holds even where $\Psi$ is not differentiable, in the sense that the edge of associated points is given by the subgradient, as follows easily from Theorem 3.3 For the Wulff shape, (5) holds in all dimensions.

Proof of Proposition 3.1. First we consider the case that $C$ is differentiable at $p_{1}$ and $p_{2}$. For any fixed vector $\mathbf{e}$, consider a variation of the form $\mathbf{v}=\varphi \mathbf{e}$, where $\varphi$ rises quickly and nicely from 0 to 1 in a small neighborhood of $p_{1}$ and falls quickly and nicely back to 0 in a small neighborhood of $p_{2}$. The directional derivative 
$D \Phi(\mathbf{e})$ is continuous at $p_{1}$ and $p_{2}$ (see Definitions 2.1). In the limit, by formula 2.1(2) plus an area term, the nonnegativity of the first variation of $\Phi-\lambda A$ yields

$$
0 \leq D \Phi\left(\mathbf{T}_{2}\right) \bullet \mathbf{e}-D \Phi\left(\mathbf{T}_{1}\right) \bullet \mathbf{e}-\lambda\left(p_{2}-p_{1}\right)^{\perp} \bullet \mathbf{e} .
$$

Therefore, since equality holds for every e, (4) follows.

Now suppose that $C$ is not differentiable at $p_{1}$, so that the one-sided tangents $\mathbf{T}_{1^{-}}, \mathbf{T}_{1^{+}}$are distinct (and $\mathbf{T}_{1}$ lies in the closed interval between them). We claim that

$$
D \Phi\left(\mathbf{T}_{1^{-}}\right)\left(\mathbf{T}_{1^{-}}\right)=D \Phi\left(\mathbf{T}_{1}\right)\left(\mathbf{T}_{1^{-}}\right) \text {and } D \Phi\left(\mathbf{T}_{1^{+}}\right)\left(\mathbf{T}_{1^{+}}\right)=D \Phi\left(\mathbf{T}_{1}\right)\left(\mathbf{T}_{1^{+}}\right) .
$$

Let $\mathbf{a}=-D \Phi\left(\mathbf{T}_{1}\right)^{\perp}$, so that $D \Phi\left(\mathbf{T}_{1}\right)(\mathbf{a})=0$. Since $\Phi$ is convex, a small distance beyond $p_{1}, D \Phi(\mathbf{T})(\mathbf{a}) \geq 0$, with equality only if $\Phi$ is linear in between. Similarly a small distance before $p_{1}, D \Phi(\mathbf{T})(\mathbf{a}) \leq 0$, with equality only if $\Phi$ is linear in between. In particular,

$$
0 \geq D \Phi\left(\mathbf{T}_{1^{-}}\right)(\mathbf{a})-D \Phi\left(\mathbf{T}_{1^{+}}\right)(\mathbf{a})
$$

with equality only if $\Phi$ is linear on the open sector from $\mathbf{T}_{1}^{-}$to $\mathbf{T}_{1}^{+}$.

Consider a variation of the form $\varphi \mathbf{a}$, where $\varphi$ rises quickly and nicely from 0 to 1 just before $p_{1}$ and falls quickly and nicely back to 0 just after $p_{1}$. In the limit the nonnegativity of the first variation yields

$$
0 \leq D \Phi\left(\mathbf{T}_{1^{-}}\right)(\mathbf{a})-D \Phi\left(\mathbf{T}_{1^{+}}\right)(\mathbf{a}) .
$$

By (7), $\Phi$ must be linear in the open interval from $\mathbf{T}_{1^{-}}$to $\mathbf{T}_{1^{+}}$, so that

$$
\left.\lim _{\mathbf{T} \rightarrow \mathbf{T}_{1^{-}}} D \Phi(\mathbf{T})\left(\mathbf{T}_{1^{-}}\right)=D \Phi\left(\mathbf{T}_{1}\right) \mathbf{T}_{1^{-}}\right), \quad \lim _{\mathbf{T} \rightarrow \mathbf{T}_{1^{+}-}} D \Phi(\mathbf{T})\left(\mathbf{T}_{1^{+}}\right)=D \Phi\left(\mathbf{T}_{1}\right)\left(\mathbf{T}_{1^{+}}\right) .
$$

Now (6) follows from the general property of norms (Definitions 2.1) that

$$
\lim _{\mathbf{T} \rightarrow \mathbf{T}_{0}} D \Phi(\mathbf{T})\left(\mathbf{T}_{0}\right)=D \Phi\left(\mathbf{T}_{0}\right)\left(\mathbf{T}_{0}\right) .
$$

Now given any fixed vector $\mathbf{e}$, write $\mathbf{e}$ as a nonnegative linear combination $\alpha_{1} \mathbf{T}_{1^{-}}+\alpha_{2} \mathbf{T}_{1^{+}}$of the one-sided tangents to $C$ at $p_{1}$. Consider a variation that changes quickly and nicely from 0 to $\alpha_{1} \mathbf{T}_{1^{-}}$in a small interval before $p_{1}$ and from $\alpha_{1} \mathbf{T}_{1^{-}}$to $\mathbf{e}$ in a small interval after $p_{1}$. In a small neighborhood of $p_{2}$, it changes back to 0 as in the first or this new construction, according to whether or not $C$ is differentiable at $p_{2}$. In the limit, by (6) and (8), the nonnegativity of the variation and its opposite yield

$$
0=D \Phi\left(\mathbf{T}_{2}\right) \bullet \mathbf{e}-D \Phi\left(\mathbf{T}_{1}\right) \bullet \mathbf{e}-\lambda\left(p_{2}-p_{1}\right)^{\perp} \bullet \mathbf{e},
$$

and (4) follows.

The following theorem is the main result of this paper.

3.3. Theorem. For any (continuous) norm on $\mathbf{R}^{2}$, an equilibrium closed connected curve $C$ must be a (scaled) Wulff shape with integer multiplicity.

Remarks. A curve of several components is in equilibrium if each component has the same generalized curvature $\lambda$.

Proof of Theorem 3.3. By Proposition 2.3 for some nonzero $\lambda$, the first variation of $\Phi-\lambda A$ vanishes for all Lipschitz variations. By scaling and reversing orientation if necessary, we may assume that $\lambda$ is the same as for the unit Wulff shape $W$. The curve $C$ must be locally embedded, or homothetically shrinking a small loop with 
$\operatorname{cost} \varepsilon$ and area at most $c \varepsilon^{2}$ (by the standard isoperimetric inequality) would have negative first variation.

Next we claim that $C$ is locally convex (turns only to the left, the side of the area bounded). Otherwise, a small embedded piece $C_{0}$ lies inside (to the left of) the line through its endpoints. Choose a norm-nonincreasing direction a of projection onto that line, and on that piece consider the variation $\varphi \mathbf{a}$, where $\varphi$ is distance from the line. Then initially $d \Phi / d t \leq 0$ while $d A / d t>0$, so that the first variation of $\Phi-\lambda A$ is negative, a contradiction. To prove that $d A / d t>0$, note that $d A / d t$ equals the flux of $\varphi \mathbf{a}$ through $C_{0}$, which in turn equals minus the integral over the region between $C_{0}$ and the line of $\operatorname{div}(\varphi \mathbf{a})$, which is positive.

Now by Proposition 3.1, except for countably many directions, the vector between points of contact with supporting lines is determined. Therefore $C$ must be the Wulff shape, possibly with multiplicity.

3.4. Unsymmetric norms. All of the results of this paper extend to "unsymmetric norms," without the usual hypothesis that $\Phi(-\mathbf{T})=\Phi(\mathbf{T})$. If the enclosed area is negative, the equilibrium curve is the central inversion of the Wulff shape. In the proof that an equilibrium curve is locally convex, to find the norm-nonincreasing projection onto a line, it may be necessary to add on to $\Phi$ a linear function, which does not alter the energy of a closed curve. This final argument was discovered while the author was visiting Marcos Salvai at the University of Córdoba, Argentina.

\section{ACKNOWLEDGMENTS}

This question emerged from email conversations with Antonio Ros while I was visiting Áladar Heppes at the Renyi Institute in Budapest, December, 2002. The work is partially supported by a National Science Foundation grant.

\section{REFERENCES}

[A] A. D. Aleksandrov, Uniqueness theorems for surfaces in the large. $V$, Vestnik Leningrad Univ. Mat. Mekh. Astronom. 13 (1958), 5-8; Amer. Math. Soc. Transl. (2) 21 (1962), 412-416. MR 27:698e

[BdC] João Lucas Barbosa and Manfredo do Carmo, Stability of hypersurfaces with constant mean curvature, Math. Z. 185 (1984), 339-353. MR85k:58021c

[K1] Nicolaos Kapouleas, Compact constant mean curvature surfaces in Euclidean three-space, J. Diff. Geom. 33 (1991), 683-715. MR.93a:53007b

[K2] Nicolaos Kapouleas, Constant mean curvature surfaces in Euclidean three-space, Bull. AMS 17 (1987), 318-326. MR88g:53013

[M1] Frank Morgan, Cylindrical surfaces of Delaunay, preprint (2003).

[M2] Frank Morgan, Hexagonal surfaces of Kapouleas, Pacific J. Math., to appear.

[M3] Frank Morgan, Riemannian Geometry: A Beginner's Guide, A K Peters, Ltd., 1998. MF 98i:53001.

[P] Bennett Palmer, Stability of the Wulff shape, Proc. AMS 126 (1998), 3661-3667. MF $99 \mathrm{~b}: 58055$

[T] Jean Taylor, Crystalline variational problems, Bull. AMS 84 (1978), 568-588. MR,58:12649

[W] Henry C. Wente, Counterexample to a conjecture of H. Hopf, Pacific J. Math. 121 (1986), 193-243. MR 87d:53013

Department of Mathematics and Statistics, Williams College, Williamstown, MASSACHUSETTS 01267

E-mail address: frank.morgan@williams.edu 